\title{
Global Laurent Expansions on Riemann Surfaces *
}

\author{
Slawomir Klimek and Andrzej Lesniewski \\ Harvard University, Cambridge, MA 02138, USA
}

\begin{abstract}
We discuss global Laurent expansions for meromorphic $h$-forms on a compact Riemann surface of genus $g \geqq 2$. Our approach is motivated by Krichever and Novikov's work on string theory.
\end{abstract}

\section{Introduction}

Krichever and Novikov [KN1,2], in their study connected to conformal field theory on a Riemann surface $S$ of arbitrary genus $g$, introduced the notion of a global Laurent expansion of a meromorphic $h$-form on $S$. Their approach consists in the following. Let $P_{0}$ and $P_{\infty}$ be two distinguished points on $S$ in general position. There exists a sequence of $h$-forms $f_{n}^{(h)}, n, h \in \mathbb{Z}$, holomorphic on $S$ except, possibly, for $P_{0}$ and $P_{\infty}$, where the orders of $f_{n}^{(h)}$ are prescribed. The forms $\left\{f_{n}^{(h)}\right\}$ serve as a basis with respect to which an $h$-form $\omega$ holomorphic in an annulus on $S$ (see Sect. II for the definition) can be expanded in a convergent series. In the case of $g=0$ these forms are given by $f_{n}^{(h)}(x)=x^{n-h}(d x)^{h}$. The special feature of these expansions is that they are formulated in a coordinate independent way. To the best of our knowledge, global expansion on a Riemann surface were first discussed in $[\mathrm{BS}]$.

The present study is concerned with a detailed analysis of the KricheverNovikov (KN) expansions. We find an explicit representation of $f_{n}^{(h)}$ in terms of the Riemann theta function and prove pointwise estimates on $f_{n}^{(h)}$. It appears that a complete proof of convergence of the $\mathrm{KN}$ expansion is impossible without this explicit form of $f_{n}^{(h)}$. In particular, crucial for the convergence is a detailed analysis of the normalization constants occurring in $f_{n}^{(h)}$. This leads to a small denominator problem which has not been discussed in $[\mathrm{KN} 1,2]$ and which is settled here. Furthermore, we define generalized Cauchy kernels $K^{(h)}(x, y)$ which serve to generate the expansion. Using Fay's trisecant identities [F] we find closed form expressions for $K^{(h)}(x, y)$. Similar representations of $f_{n}^{(h)}$ and $K^{(h)}(x, y)$ can be found in the context of conformal $b-c$ systems [S], [BLMR] (this was brought to our attention by Hidenori Sonoda); our point is also to address the analytic questions. We would like to mention that there is a relation between the $\mathrm{KN}$ approach to the chiral algebras on Riemann surfaces and the operator formalism developed in [AGMV].

* Supported in part by the Department of Energy under Grant DE-FG02-88ER25065 
The paper is organized as follows. In Sect. II we review briefly basic facts about Riemann surfaces and the theta function which are relevant for our study. Section III contains the construction of the KN basis $f_{n}^{(h)}$. In Sect. IV we define and study the Cauchy kernels $K^{(h)}(x, y)$. Section IV is of technical character and deals with the small denominators of $f_{n}^{(h)}$. Finally, in Sect. IV we describe $n$-dimensional generalizations of the $\mathrm{KN}$ expansion.

\section{Basic Definitions}

Let $S$ be a compact Riemann surface of genus $g \geqq 2$ with a fixed canonical homology basis $\left\{a_{1}, \ldots, a_{g}, b_{1}, \ldots, b_{g}\right\}$. Let $(I, \tau)$ be the corresponding period matrix. Recall that $\tau$ is a symmetric $g \times g$ matrix with $\operatorname{Im} \tau>0$. By $\operatorname{Div}(S)$ we denote the (additive) group of divisors on $S$. Let $\phi: \operatorname{Div}(S) \rightarrow J(S)$ be the Jacobi map with respect to a fixed base point, where $J(S)$ is the Jacobi variety. Let $\theta(\xi), \xi \in \mathbb{C}^{g}$ be the Riemann theta function. By $\theta[\alpha](\xi)$ we denote the Riemann theta function with integer characteristics $\alpha=\left(\begin{array}{c}\varepsilon_{1}, \ldots, \varepsilon_{g} \\ \varepsilon_{1}^{\prime}, \ldots, \varepsilon_{g}^{\prime}\end{array}\right) \varepsilon_{j}, \varepsilon_{j}^{\prime} \in \mathbb{Z}$. Recall that each integer theta characteristic $\alpha$ determines a spin bundle $L_{\alpha}$ on $S$ according to

$$
\phi\left(\text { divisor of } L_{\alpha}\right)=-\frac{\tau \varepsilon}{2}-\frac{\varepsilon^{\prime}}{2}-K
$$

where $K=\phi(\Delta)$ is the vector of Riemann's constants.

Let $\mathcal{O}\left(\equiv \mathcal{O}^{0}\right)$ be the sheaf of meromorphic functions on $S$ and let $\mathcal{O}^{1}$ be the sheaf of meromorphic 1-forms. By $\mathcal{O}^{h}:=\left(\mathcal{O}^{1}\right)^{\otimes h}, h \in \mathbb{Z} \backslash\{0\}$, we denote the sheaf of meromorphic $h$-forms. Furthermore, let $\Omega^{h}, h \geqq 1$, be the sheaf of holomorphic $h$-forms. For a sheaf $\mathscr{F}$ on $S$, we denote by $\Gamma(S, \mathscr{F})$ the space of its sections. There is a natural way to define pointwise convergence on $\Gamma\left(S, \mathcal{O}^{h}\right)$. We choose a Riemannian metric $\gamma$ on $S$. For definiteness we take

$$
\gamma=\sum_{j=1}^{g}\left(\bar{\zeta}_{j} \otimes \zeta_{j}+\zeta_{j} \otimes \bar{\zeta}_{j}\right)
$$

with $\zeta_{1}(x), \ldots, \zeta_{g}(x)$ the canonical basis of $\Gamma\left(S, \Omega^{1}\right)$ associated with $\left\{a_{1}, \ldots, a_{g}, b_{1}, \ldots, b_{g}\right\}$. Note that $\gamma$ is the pull-back of the canonical flat metric on $J(S)$ via $\phi$. The absolute value of $\omega \in \Gamma\left(S, \mathcal{O}^{h}\right)$ at $x \in S$ is given by

$$
|\omega(x)|:=\left\{\overline{\omega(x)} \omega(x) \gamma(x)^{-h}\right\}^{1 / 2} .
$$

Clearly, $|\omega(x)|$ is independent of the choice of coordinates at $x$. The absolute value $|\omega(x)|$ defines the notion of pointwise convergence of series of meromorphic $h$-forms on $S$.

In order to find a suitable generalization of a circle and an annulus on $S$ we adopt an idea of [KN1]. Let $P_{0}$ and $P_{\infty}$ be two points on $S$ in general position (in particular, $P_{0}$ and $P_{\infty}$ are no $h$-Weierstrass points). Given $P_{0}$ and $P_{\infty}$ there is a unique $\eta_{P_{0}-P_{\infty}} \in \Gamma\left(S, \mathcal{O}^{1}\right)$ such that: (i) it has simple poles at $P_{0}$ and $P_{\infty}$ and no other poles, (ii) $\operatorname{Res}_{P_{0}} \eta_{P_{0}-P_{\infty}}(x)=1$, (iii) $\operatorname{Re} \int_{a_{J}} \eta_{P_{0}-P_{\infty}}=\operatorname{Re} \int_{b_{j}} \eta_{P_{0}-P_{\infty}}=0$. We choose a base point $P_{1}, P_{1} \neq P_{0}, P_{1} \neq P_{\infty}$, and set 


$$
R(x):=\exp \left\{\operatorname{Re} \int_{P_{1}}^{x} \eta_{P_{0}-P_{\infty}}\right\} .
$$

Our definition implies that $R(\mathrm{x})$ is a well-defined function on $S$. Furthermore, $R\left(P_{0}\right)=0, R\left(P_{1}\right)=1$ and $R\left(P_{\infty}\right)=\infty$. This function will play the role similar to that of the absolute value $|x|$ on $\mathbb{C P}^{1}$.

The cycle

$$
\gamma(r):=\{x \in S: R(x)=r\}, \quad 0<r<\infty,
$$

is called a circle of radius $r$. The set

$$
D(r):=\{x \in S: R(x)<r\}
$$

is called an (open) disc of radius $r$. For $R>r>0$ the set

$$
A(r ; R)=D_{R} \backslash \bar{D}_{r}
$$

is called an (open) annulus of radii $r$ and $R$.

It is remarkable that $R(x)$ is in fact a proper generalization of $|x|$. For example, the following proposition extends the classical Hadamard three circles theorem.

Proposition II.1. Let $f$ be a function holomorphic in an annulus $A(r ; R)$. For $r<\rho<R$ we set

$$
M(\rho):=\max _{x: R(x)=\rho} \log |f(x)| .
$$

Then for $r<r_{1}<r_{2}<r_{3}<R$,

$$
M\left(r_{2}\right) \leqq M\left(r_{1}\right) \frac{\log r_{3}-\log r_{2}}{\log r_{3}-\log r_{1}}+M\left(r_{3}\right) \frac{\log r_{2}-\log r_{1}}{\log r_{3}-\log r_{1}} .
$$

Proof. The function $\log R(x)=\operatorname{Re} \int_{P_{1}}^{x} \eta_{P_{0}-P_{\infty}}$ is harmonic and thus obeys the maximum principle. The proof of the classical three circles theorem (see e.g., $[\mathrm{HCR}]$ ) immediately generalizes to our setting.

Of fundamental importance for our study is the prime form on $S$ ([F], [M]). We review briefly its definition and main properties referring the reader to $[\mathrm{F}],[\mathrm{M}]$ for the details.

Let $\alpha$ be a nonsingular odd theta characteristic, i.e., $\alpha$ is integer and $\xi=0$ is a first-order zero of $\theta[\alpha](\xi)$. There is a section $h_{\alpha}(x)$ of $L_{\alpha}$ such that

$$
h_{\alpha}(x)^{2}=\sum_{j=1}^{g} \partial_{j} \theta[\alpha](0) \zeta_{j}(x) .
$$

The prime form $E(x, y)$ is defined by

$$
E(x, y):=\frac{\theta[\alpha](\phi(y-x))}{h_{\alpha}(x) h_{\alpha}(y)} .
$$

One can show that $E(x, y)$ is independent of the choice of $\alpha$. Moreover, $E(x, y)$ is antisymmetric,

$$
E(x, y)=-E(y, x)
$$


and $x=y$ is its only (simple) zero. In local coordinates $t$ around $x$ such that $d t(x)=h_{\alpha}(x)^{2}$ we have for $y$ close to $x$

$$
E(x, y)=\frac{t(y)-t(x)}{\sqrt{d t(x)} \sqrt{d t(y)}}\left(1+O\left((t(x)-t(y))^{2}\right) .\right.
$$

For a fixed $x, E(x, y)$ defines a multivalued holomorphic differential form of weight $-1 / 2$ in $y$ with multipliers along the $a_{j}$ and $b_{j}$ cycles given by

$$
\begin{gathered}
\chi\left(a_{j}\right)=1, \\
\chi\left(b_{j}\right)=\exp 2 \pi i\left(-\frac{1}{2} \tau_{j j}-\phi_{j}(y-x)\right) .
\end{gathered}
$$

The prime form $E(x, y)$ provides a tool to construct meromorphic forms on $S$. For example, for

$$
D=\sum P_{j}-\sum Q_{j} \in \operatorname{Div}(S)
$$

such that $\phi(D)=0$, we can explicitly write any $f$ with $(f)=D$ :

$$
f(x)=\text { const. } \prod E\left(x_{j}, P_{j}\right) / \prod E\left(x, Q_{j}\right) .
$$

Also, the form $\eta_{P_{0}-P_{\infty}}$ can be expressed in terms of $E$. We set

$$
\omega_{P-Q}(x)=d \log \frac{E(x, P)}{E(x, Q)} .
$$

Then $\eta_{P_{0}-P_{\infty}}$ is given by

$$
\eta_{P_{0}-P_{\infty}}(x)=\omega_{P_{0}-P_{\infty}}(x)+i \sum_{j, k}\left((\operatorname{Im} \tau)^{-1}\right)_{j k}\left(\operatorname{Re} \int_{b_{j}} \omega_{P_{0}-P_{\infty}}\right) \zeta_{k}(x) .
$$

There is a number of useful identities involving the prime form and the theta function. The most fundamental is Fay's trisecant identity [F]. Let

$$
\vartheta(x):=\theta \circ \phi(x) \text {. }
$$

Then for $x, y, P, Q \in S$ and $Z \in \operatorname{Div}(S)$,

$$
\begin{aligned}
\vartheta(Z) & \vartheta(Z+P+Q-x-y) E(P, Q) E(x, y) \\
= & -\vartheta(Z+P-x) \vartheta(Z+Q-y) E(y, P) E(x, Q) \\
& +\vartheta(Z+P-y) \vartheta(Z+Q-x) E(x, P) E(y, Q) .
\end{aligned}
$$

Another useful identity is $[\mathrm{F}]$ :

$$
\begin{aligned}
& \frac{\vartheta(x-P+Z) \vartheta(x-Q-Z)}{\vartheta(Z) \vartheta(Z+Q-P)} \frac{E(P, Q)}{E(x, P) E(x, Q)} \\
& =\omega_{Q-P}(x)+\sum_{j=1}^{g}\left\{\partial_{j} \log \vartheta(Z+Q-P)-\partial_{j} \log \vartheta(Z)\right\} \zeta_{j}(x) .
\end{aligned}
$$

We shall also need the following (multivalued) $g / 2$-form $\sigma(x)[\mathrm{F}]$ :

$$
\sigma(x)=\exp \left\{-\sum_{j=1}^{g} \int_{a_{j}} \zeta_{j}(y) \log E(y, x)\right\} .
$$


Note that $\sigma(x)$ has no zeroes and poles on $S$. Its multipliers along $a_{j}$ and $b_{j}$ are

$$
\begin{gathered}
\chi\left(a_{j}\right)=1, \\
\chi\left(b_{j}\right)=\exp 2 \pi i\left\{\frac{1}{2}(g-1) \tau_{j j}+(g-1) \phi_{j}(x)-K_{j}\right\} .
\end{gathered}
$$

The following identity involving $\sigma$ will also be useful later. For generic $P$,

$$
\frac{\sigma(x)}{\sigma(y)}\left(\frac{E(x, P)}{E(y, P)}\right)^{g}=\frac{\vartheta(x-g P-\Delta)}{\vartheta(y-g P-\Delta)} .
$$

\section{The Krichever-Novikov Basis}

In this section we prove the existence and construct a Krichever-Novikov (KN) basis for meromorphic $h$-forms with possible poles at $P_{0}$ and $P_{\infty}$. We consider a sequence of divisors

$$
D_{n}^{(h)}=k_{n}^{(h)} P_{0}+m_{n}^{(h)} P_{\infty}
$$

which describe the behavior of the elements of a $\mathrm{KN}$ basis at $P_{0}$ and $P_{\infty}$. Specifically,

$$
D_{n}^{(h)}= \begin{cases}0, & \text { if } h=0, n=0, \\ n P_{0}-(n+g+1) P_{\infty}, & \text { if } h=0, \quad-g \leqq n \leqq-1, \\ -P_{0}-P_{\infty}, & \text { if } h=1, \quad n=0, \\ (n-1) P_{0}-(n-g) P_{\infty}, & \text { if } h=1, \quad 1 \leqq n \leqq g, \\ (n-h) P_{0}-(n+h-(2 h-1) g) P_{\infty}, & \text { otherwise }\end{cases}
$$

The theorem below asserts that $h$-forms with the prescribed orders of zeroes and poles at $P_{0}$ and $P_{\infty}$ exist and are essentially unique (except for $h=0$, $-g \leqq n \leqq-1$ and $h=1, n=0)$. For $D \in \operatorname{Div}(S)$ we write $\mathcal{O}_{D}^{h}$ to denote the sheaf of meromorphic $h$-forms $\omega$ with $(\omega) \geqq D$.

Theorem III.1. (i) Let $(h, n) \notin\{(0,-g), \ldots,(0,-1),(1,0)\}$. Then there is a unique (up to a constant factor) $f_{n}^{(h)} \in \Gamma\left(S, \mathcal{O}_{D_{n}^{h}}^{(h)}\right)$ such that

$$
\operatorname{ord}_{P_{0}} f_{n}^{(h)}=k_{n}^{(h)}, \quad \operatorname{ord}_{P_{\infty}} f_{n}^{(h)}=m_{n}^{(h)} .
$$

(ii) For $h=0,-g \leqq n \leqq-1$ there are two linearly independent $f_{n}^{(0)}, g_{n}^{(0)} \in \Gamma\left(S, \mathcal{O}_{D_{n}^{(0)}}\right)$ such that (III.3) holds. Furthermore,

$$
g_{n}^{(0)}=\alpha_{n} f_{n}^{(0)}+\beta_{n}, \quad \alpha_{n}, \beta_{n} \in \mathbb{C}, \quad \alpha_{n} \neq 0 .
$$

(iii) For $h=1, n=0$, there are $g+1$ linearly independent $f_{0}^{(1)} \in \Gamma\left(S, \mathcal{O}_{D_{0}^{(1)}}\right)$ such that (III.3) holds. Each of them can be written as

$$
f_{0}^{(1)}=\gamma_{0} \omega_{P_{0}-P_{\infty}}+\sum_{j=1}^{g} \gamma_{j} \zeta_{j}, \quad \gamma_{j} \in \mathbb{C}, \quad \gamma_{0} \neq 0
$$

Proof. Let us first assume that $h \geqq 2$, and let $P \in S$ be non-Weierstrass. We claim that

$$
\operatorname{dim} \Gamma\left(S, \mathcal{O}_{k P}^{h}\right)=\max \left\{d_{h}-k, 0\right\},
$$

where $d_{h}=\operatorname{dim} \Gamma\left(S, \Omega^{h}\right)=(2 h-1)(g-1)$. In fact, for $k \geqq 0$ (III.6) follows from the 
gap theorem. For $k<0$ we use the Riemann-Roch theorem and Serre's duality:

$$
\operatorname{dim} \Gamma\left(S, \mathcal{O}_{k P}^{h}\right)=d_{h}-k+\operatorname{dim} \Gamma\left(S, \mathcal{O}_{-k P}^{1-h}\right) .
$$

Since $\operatorname{dim} \Gamma\left(S, \Omega^{1-h}\right)=0$ and $-k>0$, it follows that $\operatorname{dim} \Gamma\left(S, \mathcal{O}_{-k P}^{1-h}\right)=0$ and (III.6) is proved. Take now $P=P_{0}$ and $k=n-h, n<d_{h}+h$. It follows from (III.6) that $\operatorname{dim} \Gamma\left(S, \mathcal{O}_{(n-h) P_{0}}^{h}\right)=d_{h}-n+h$ and by linear algebra there is precisely one (up to a constant factor) $\omega \in \Gamma\left(S, \mathcal{O}_{(n-h) P_{0}}^{h}\right)$ with a zero of order $d_{h}-n+h-1=$ $-(n+h-(2 h-1) g)$ at $P_{\infty}$. This proves our assertion for $n<d_{h}+h$. If $n \geqq d_{h}+h$, we use (III.6) with $P=P_{\infty}, k=d_{h}-n+h-1$.

We assume now that $h=1$. The statement about $n=0$ is well-known. For $n \notin\{0,1, \ldots, g\}$ we use (III.6) with $P=P_{0}, k=n-1$ (if $n<g$ ) or $P=P_{\infty}, k=g-n-1$ (if $n \geqq g$ ). If $1 \leqq n \leqq g$, we note that $\operatorname{dim} \Gamma\left(S, \mathcal{O}_{(n-1) P_{0}}\right)=g-n+1$ and, by linear algebra, there is precisely one (up to a constant factor) 1-form with a zero of order $g-n$ at $P_{\infty}$.

The proof for $h \leqq 0$ is similar and we omit the details.

We can partially remove the nonuniqueness of $f_{n}^{(h)}$ by imposing the following condition:

$$
\operatorname{Res}_{P_{0}} f_{n}^{(1-h)} f_{m}^{(h)}=\delta_{n+m, 0} .
$$

It is clear that such a choice is possible. It fixes $f_{n}^{(h)}, h \leqq 0$, completely and leaves a freedom of choice of a constant factor in $f_{n}^{(h)}, h \geqq 1$, and of $f_{0}^{(1)}$.

Definition III.2. A sequence of meromorphic $h$-forms $f_{n}^{(h)}, n \in \mathbb{Z}$, given by Theorem III.1 and obeying (III.8) is called a KN basis.

We now introduce explicit formulas for $f_{n}^{(h)}(x)$. We set

$$
f_{n}^{(0)}(x)= \begin{cases}\sigma(x)^{-1} \frac{E\left(x, P_{0}\right)^{n}}{E\left(x, P_{\infty}\right)^{n+g}} \frac{\vartheta\left(x+D_{n}^{(0)}-\Delta\right)}{\vartheta\left(P_{0}+D_{n}^{(0)}-\Delta\right)}, & \text { if } n \notin\{-g, \ldots,-1,0\}, \\ 1, & \text { if } n=0 \\ \sigma(x)^{-1} \frac{E\left(x, p_{0}\right)^{n} E\left(x, P_{1}\right)}{E\left(x, P_{\infty}\right)^{n+g+1}} \frac{\vartheta\left(x+P_{1}+D_{n}^{(0)}-\Delta\right)}{\vartheta\left(P_{0}+P_{1}+D_{n}^{(0)}-\Delta\right)}, & \text { if } \quad-g \leqq n \leqq-1,\end{cases}
$$

$$
f_{n}^{(1)}(x)=\left\{\begin{array}{cc}
E\left(P_{0}, P_{\infty}\right) \sigma(x) \frac{E\left(x, P_{0}\right)^{n-1}}{E\left(x, P_{\infty}\right)^{n-g+1}} \frac{\vartheta\left(x+D_{n}^{(1)}+\Delta\right)}{\vartheta\left(P_{0}+D_{n}^{(1)}+\Delta\right)}, & \text { if } n \notin\{0,1, \ldots, g\}, \\
\frac{E\left(P_{0}, P_{1}\right)}{E\left(x, P_{0}\right) E\left(x, P_{1}\right)} \frac{\vartheta\left(x+g P_{\infty}-P_{0}-P_{1}+\Delta\right)}{\vartheta\left(g P_{\infty}-P_{0}+\Delta\right)} \frac{\vartheta\left(x-g P_{\infty}-\Delta\right)}{\vartheta\left(P_{1}-g P_{\infty}-\Delta\right)} \\
-\frac{E\left(P_{\infty}, P_{1}\right)}{E\left(x, P_{\infty}\right) E\left(x, P_{1}\right)} \frac{\vartheta\left(x+g P_{0}-P_{\infty}-P_{1}+\Delta\right)}{\vartheta\left(g P_{0}-P_{\infty}+\Delta\right)} \frac{\vartheta\left(x-g P_{0}-\Delta\right)}{\vartheta\left(P_{1}-g P_{0}-\Delta\right)}, \\
\text { if } \quad n=0, \\
E\left(P_{0}, P_{\infty}\right) \sigma(x) \frac{E\left(x, P_{0}\right)^{n-1}}{E\left(x, P_{\infty}\right)^{n-g} E\left(x, P_{1}\right)} \frac{\vartheta\left(x-P_{1}+D_{n}^{(1)}+\Delta\right)}{\vartheta\left(P_{0}-P_{1}+D_{n}^{(1)}+\Delta\right)}, \\
\text { if } \quad 1 \leqq n \leqq g,
\end{array}\right.
$$




$$
\begin{gathered}
f_{n}^{(h)}(x)=E\left(P_{0}, P_{\infty}\right)^{(\operatorname{sign} h+1) / 2} \sigma(x)^{2 h-1}, \\
\frac{E\left(x, P_{0}\right)^{n-h}}{E\left(x, P_{\infty}\right)^{(n+h-2 h g+g)}} \frac{\vartheta\left(x+D_{n}^{(h)}+(2 h-1) \Delta\right)}{\vartheta\left(P_{0}+D_{n}^{(h)}+(2 h-1) \Delta\right)}, \quad h \neq 0,1 .
\end{gathered}
$$

Remarks. 1. The choice of $P_{1}$ in (III.9) and (III.10) is optional. It could be replaced by any other generic point of $S$.

2. The singularities of $f_{0}^{(1)}$ at $x=P_{1}$ are removable. The coefficients have been chosen in such a way that both poles cancel.

Theorem III.3. For each $h \in \mathbb{Z}$, the set $\left\{f_{n}^{(h)}\right\}_{n \in \mathbb{Z}}$ is a KN basis. Furthermore, there exist constants $A_{h}, B, \varepsilon>0$ such that

where

$$
\left|f_{n}^{(h)}\right| \leqq \begin{cases}A_{0}^{(n)}(B R(x))^{n}(1+B R(x))^{g+1}, & h=0, \quad-g \leqq n \leqq-1 \\ A_{1}^{(n)}(B R(x))^{n-1}(1+B R(x))^{-g+1}, & h=1, \quad 1 \leqq n \leqq g \\ A_{1}^{(n)}(B R(x))^{-1}(1+B R(x))^{2}, & h=1, \quad n=0 \\ 1, & h=0, \quad n=0 \\ A_{h}^{(n)}(B R(x))^{n-h}(1+B R(x))^{2 h-2 h g+g}, & \text { otherwise; }\end{cases}
$$

Proof. We prove the first assertion. The proof of (III.12) requires solving a small denominators problem and we defer it to Sect. V.

We have to verify that $f_{n}^{(h)}(x)$ are single-valued and that they obey (III.8). It is clear that they have correct zeroes and poles at $P_{0}$ and $P_{\infty}$. Assume that $n \neq\{-g, \ldots,-1,0\}$, if $h=0$ and that $n \neq\{0,1, \ldots, g\}$, if $h=1$. Then each $f_{n}^{(h)}(x)$ has additional zeroes $R_{1}, \ldots, R_{g}$ except for those prescribed by the divisor $D_{n}^{(h)}$ (note that $R_{1}, \ldots, R_{g}$ depend on $h$ and $n$ ). Then

$$
f_{n}^{(h)}(x)=C_{n}^{(h)} \sigma(x)^{2 h-1} \frac{E\left(x, P_{0}\right)^{n-h}}{E\left(x, P_{\infty}\right)^{n+h-2 h g+g}} \vartheta\left(x-\sum_{j=1}^{g} R_{j}-\Delta\right),
$$

where $C_{n}^{(h)}$ is a constant, is a well-defined $h$-form. Using the fact that

$$
\phi\left(D_{n}^{(h)}+\sum_{j=1}^{g} R_{j}\right)=-2 h K
$$

we arrive at (III.9-11).

Let $h=0,-g \leqq n \leqq-1$. Then for $f_{n}^{(0)}(x)$ has $g+1$ zeroes $R_{1}, \ldots, R_{g+1}$ except for those prescribed by $D_{n}^{(0)}$. By means of Theorem III.1(ii) we can choose $R_{g+1}=P_{1}$ and then repeat the above argument.

Similarly we show that $f_{n}^{(1)}(x)$ is well-defined for $0 \leqq n \leqq g$.

To verify (III.8) we notice that the only nonobvious issue is to show that

$$
\operatorname{Res}_{P_{0}} f_{n}^{(0)}(x) f_{0}^{(1)}(x)=0, \quad-g \leqq n \leqq-1 .
$$

We write $f_{0}^{(1)}(x)=\omega_{1}(x)-\omega_{2}(x)$, in a self-explanatory notation. Using (II.25) with $y=P_{1}, P=P_{\infty}$, we obtain

$$
\omega_{1}(x)=\frac{\sigma(x)}{\sigma\left(P_{1}\right)} \frac{E\left(x, P_{\infty}\right)^{g}}{E\left(P_{1}, P_{\infty}\right)^{g}} \frac{E\left(P_{0}, P_{1}\right)}{E\left(x, P_{0}\right) E\left(x, P_{1}\right)} \frac{\vartheta\left(x+g P_{\infty}-P_{0}-P_{1}+\Delta\right)}{\vartheta\left(g P_{\infty}-P_{0}+\Delta\right)} .
$$


Hence, $f_{n}^{(0)}(x) \omega_{1}(x)(-g \leqq n \leqq-1)$ is regular everywhere except for $x=P_{0}$ and thus $\operatorname{Res}_{P_{0}} f_{n}^{(0)}(x) \omega_{1}(x)=0$. Similarly, using (II.25) with $y=P_{1}, P=P_{0}$, we show that

$$
\operatorname{Res}_{P_{0}} f_{n}^{(0)}(x) \omega_{2}(x)=-\operatorname{Res}_{P_{\infty}} f_{n}^{(0)}(x) \omega_{2}(x)=0
$$

\section{The Cauchy Kernels}

We set for $h \in \mathbb{Z}$

$$
K^{(h)}(x, y)=\left\{\begin{array}{lll}
\sum_{n=0}^{\infty} f_{n}^{(h)}(x) f_{-n}^{(1-h)}(y), & \text { if } & 0<R(x)<R(y)<\infty, \\
-\sum_{n=-\infty}^{-1} f_{n}^{(h)}(x) f_{-n}^{(1-h)}(y), & \text { if } & 0<R(y)<R(x)<\infty .
\end{array}\right.
$$

This definition is meaningful as a consequence of the following

Theorem IV.1. The series defining $K^{(h)}(x, y)$ converge almost uniformly in their domains. Consequently, $K^{(h)}(x, y)$ is holomorphic in $x$ and $y$ for $R(x) / R(y) \neq 0,1, \infty$. Proof. Let $n \notin\{-g, \ldots,-1,0)$, if $h=0$ and $n \notin\{0,1, \ldots, g\}$ if $h=1$. Then using (III.12) we find that

$$
\begin{aligned}
& \sum_{n}\left|f_{n}^{(h)}(x) f_{-n}^{(1-h)}(y)\right| \\
& \quad \leqq A_{h} A_{1-h} \frac{R(y)^{h-1}}{B R(x)^{h}} \frac{(1+B R(y))^{2 h g-g-2 h+2}}{(1+B R(x))^{-2 h g+2 h+g}} \sum_{n} n^{2\left(2 g^{2}+g+\varepsilon\right)}\left(\frac{R(x)}{R(y)}\right)^{n}<\infty .
\end{aligned}
$$

Similarly we prove the convergence of the second series in (IV.1). The excluded cases for $h=0$ and $h=1$ do not spoil the convergence as they affect only finitely many terms.

The following theorem provides an explicit expression for $K^{(h)}(x, y)$.

Theorem IV.2. Let $R(x) / R(y) \neq 0,1, \infty$. Then

$$
\begin{aligned}
K^{(0)}(x, y)= & \omega_{P_{\infty}-x}(y)+\sum_{j=1}^{g}\left\{\partial_{j} \log \vartheta\left(-x+g P_{\infty}+\Delta\right)-\partial_{j} \log \vartheta\left(-P_{1}+g P_{\infty}+\Delta\right)\right\} \zeta_{j}(y) \\
& +\sum_{j=1}^{g}\left\{\partial_{j} \log \vartheta\left(-P_{1}+g P_{0}+\Delta\right)-\partial_{j} \log \vartheta\left(-P_{\infty}+g P_{0}+\Delta\right)\right\} \zeta_{j}(y), \\
K^{(1)}(x, y)=- & \omega_{P_{0}-y}(x)-\sum_{j=1}^{g}\left\{\partial_{j} \log \vartheta\left(-y+g P_{\infty}+\Delta\right)-\partial_{j} \log \vartheta\left(-P_{0}+g P_{\infty}+\Delta\right)\right\} \zeta_{j}(x), \\
K^{(1-h)}(x, y)= & \frac{E\left(y, P_{0}\right)}{E\left(x, P_{0}\right) E(y, x)}\left(\frac{E\left(x, P_{0}\right) E\left(x, P_{\infty}\right)}{E\left(y, P_{0}\right) E\left(y, P_{\infty}\right)}\right)^{h} \\
& \cdot \frac{\vartheta\left(x-y+D_{0}^{(1-h)}+P_{\infty}-(2 h-1) \Delta\right) \vartheta\left(y-g P_{\infty}-\Delta\right)^{2 h-1}}{\vartheta\left(D_{0}^{(1-h)}+P_{\infty}-(2 h-1) \Delta\right) \vartheta\left(x-g P_{\infty}-\Delta\right)^{2 h-1}}, h \neq 0,1 .
\end{aligned}
$$


Proof. We start with $h \neq 0,1$. For $0<R(x)<R(y)<\infty$ we have

$$
\begin{aligned}
K^{(1-h)}(x, y)= & E\left(P_{0}, P_{\infty}\right)\left(\frac{\sigma(y)}{\sigma(x)}\right)^{2 h-1}\left(\frac{E\left(x, P_{0}\right)}{E\left(y, P_{0}\right)}\right)^{h}\left(\frac{E\left(y, P_{\infty}\right)}{E\left(x, P_{\infty}\right)}\right)^{(2 h-1) g-h} \\
& \cdot \frac{1}{E\left(x, P_{0}\right) E\left(x, P_{\infty}\right)} \sum_{n \geqq 0}\left(\frac{E\left(x, P_{0}\right) E\left(y, P_{\infty}\right)}{E\left(x, P_{\infty}\right) E\left(y, P_{0}\right)}\right)^{n} \\
& \cdot \frac{\vartheta\left(x+D_{n}^{(1-h)}-(2 h-1) \Delta\right) \vartheta\left(-y-D_{-n}^{(h)}-(2 h-1) \Delta\right)}{\vartheta\left(P_{0}+D_{n}^{(1-h)}-(2 h-1) \Delta\right) \vartheta\left(-P_{0}-D_{-n}^{(h)}-(2 h-1) \Delta\right)} .
\end{aligned}
$$

We use now the trisecant identity with $Z=x+D_{n}^{(1-h)}-(2 h-1) \Delta, a=P_{0}, b=P_{\infty}$ :

$$
\begin{aligned}
& \vartheta\left(x+D_{n}^{(1-h)}-(2 h-1) \Delta\right) \vartheta\left(-y-D_{-n}^{(h)}-(2 h-1) \Delta\right)=\frac{1}{E\left(P_{0}, P_{\infty}\right) E(x, y)} \\
& \cdot\left\{-\vartheta\left(P_{0}+D_{n}^{(1-h)}-(2 h-1) \Delta\right) \vartheta\left(x-y+D_{n}^{(1-h)}+P_{\infty}-(2 h-1) \Delta\right)\right. \\
& \cdot E\left(y, P_{0}\right) E\left(x, P_{\infty}\right)+\vartheta\left(D_{n}^{(1-h)}+P_{\infty}-(2 h-1) \Delta\right) \\
& \left.\cdot \vartheta\left(x-y+D_{n}^{(1-h)}+P_{0}-(2 h-1) \Delta\right) E\left(x, P_{0}\right) E\left(y, P_{\infty}\right)\right\} .
\end{aligned}
$$

Noting that

and

$$
D_{n}^{(1-h)}+P_{\infty}=-P_{0}-D_{-n}^{(h)}
$$

$$
D_{n+1}^{(1-h)}+P_{\infty}=D_{n}^{(1-h)}+P_{0} .
$$

We can write the sum (IV.5) as

$$
\begin{aligned}
& \frac{1}{E\left(P_{0}, P_{\infty}\right) E(x, y)}\left\{-\sum_{n \geqq 0} \frac{\left(E\left(x, P_{0}\right) E\left(y, P_{\infty}\right)\right)^{n}}{\left(E\left(x, P_{\infty}\right) E\left(y, P_{0}\right)\right)^{n-1}} \frac{\vartheta\left(x-y+D_{n}^{(1-h)}+P_{\infty}-(2 h-1) \Delta\right)}{\vartheta\left(D_{n}^{(1-h)}+P_{\infty}-(2 h-1) \Delta\right)}\right. \\
& \left.\quad+\sum_{n \geqq 0} \frac{\left(E\left(x, P_{0}\right) E\left(y, P_{\infty}\right)\right)^{n+1}}{\left(E\left(x, P_{\infty}\right) E\left(y, P_{0}\right)\right)^{n}} \frac{\vartheta\left(x-y+D_{n+1}^{(1-h)}+P_{\infty}-(2 h-1) \Delta\right)}{\vartheta\left(D_{n+1}^{(1-h)}+P_{\infty}-(2 h-1) \Delta\right)}\right\} \\
& =-\frac{E\left(x, P_{\infty}\right) E\left(y, P_{0}\right)}{E\left(P_{0}, P_{\infty}\right) E(x, y)} \frac{\vartheta\left(x-y+D_{1}^{(1-h)}+P_{\infty}-(2 h-1) \Delta\right)}{\vartheta\left(D_{1}^{(1-h)}+P_{\infty}-(2 h-1) \Delta\right)} .
\end{aligned}
$$

Substituting this into (IV.5) yields

$$
\begin{aligned}
K^{(1-h)}(x, y)= & \left(\frac{\sigma(y)}{\sigma(x)}\right)^{2 h-1}\left(\frac{E\left(x, P_{0}\right)}{E\left(y, P_{0}\right)}\right)^{h}\left(\frac{E\left(y, P_{\infty}\right)}{E\left(x, P_{\infty}\right)}\right)^{(2 h-1) g-h} \\
& \cdot \frac{E\left(y, P_{0}\right)}{E\left(x, P_{0}\right) E(y, x)} \frac{\vartheta\left(x-y+D_{n}^{(1-h)}+P_{\infty}-(2 h-1) \Delta\right)}{\vartheta\left(D_{0}^{(1-h)}+P_{\infty}-(2 h-1) \Delta\right)} .
\end{aligned}
$$

Using (II.22) with $x \rightarrow y, y \rightarrow x, P \rightarrow P_{\infty}$ yields

$$
\begin{aligned}
K^{(1-h)}(x, y)= & \left(\frac{E\left(x, P_{0}\right) E\left(x, P_{\infty}\right)}{E\left(y, P_{0}\right) E\left(y, P_{\infty}\right)}\right)^{h} \frac{E\left(y, P_{0}\right)}{E\left(x, P_{0}\right) E(y, x)} \\
& \cdot \frac{\vartheta\left(x-y+D_{0}^{(1-h)}+P_{\infty}-(2 h-1) \Delta\right) \vartheta\left(y-g P_{\infty}-\Delta\right)^{2 h-1}}{\vartheta\left(D_{0}^{(1-h)}+P_{\infty}-(2 h-1) \Delta\right) \vartheta\left(x-g P_{\infty}-\Delta\right)^{2 h-1}},
\end{aligned}
$$

as claimed. The computation for $0<R(y)<R(x)<\infty$ is analogous. 
Let $h=0,0<R(x)<R(y)<\infty$. Repeating the steps of the above computation we find that

$$
\sum_{n=1}^{\infty} f_{n}^{(0)}(x) f_{-n}^{(1)}(y)=\frac{E\left(x, P_{0}\right)}{E\left(y, P_{0}\right) E(y, x)} \frac{\vartheta\left(x-y+D_{1}^{(0)}+P_{\infty}-\Delta\right)}{\vartheta\left(D_{1}^{(0)}+P_{\infty}-\Delta\right)} \frac{\vartheta\left(y-g P_{\infty}-\Delta\right)}{\vartheta\left(x-g P_{\infty}-\Delta\right)},
$$

which by means of (II.22) equals

$$
\omega_{P_{0}-x}(y)+\sum_{j=1}^{g}\left\{\partial_{j} \log \vartheta\left(-x+g P_{\infty}+\Delta\right)-\partial_{j} \log \vartheta\left(-P_{0}+g P_{\infty}+\Delta\right)\right\} \zeta_{j}(y) .
$$

By means of the same identity,

$$
\begin{aligned}
f_{0}^{(1)}(y)= & -\omega_{P_{0}-P_{1}}(y)-\sum_{j=1}^{g}\left\{\partial_{j} \log \mathscr{Y}\left(-P_{1}+g P_{\infty}+\Delta\right)-\partial_{j} \log \mathscr{Y}\left(-P_{0}+g P_{\infty}+\Delta\right)\right\} \zeta_{j}(y) \\
& +\omega_{P_{\infty}-P_{1}}(y)+\sum_{j=1}^{g}\left\{\partial_{j} \log \mathscr{Y}\left(-P_{1}+g P_{0}+\Delta\right)-\partial_{j} \log \mathscr{Y}\left(-P_{\infty}+g P_{0}+\Delta\right)\right\} \zeta_{j}(y),
\end{aligned}
$$

and consequently,

$$
\begin{aligned}
& K^{(0)}(x, y) \\
& =\omega_{P_{\infty}-x}(y)+\sum_{j=1}^{g}\left\{\partial_{j} \log \vartheta\left(-x+g P_{\infty}+\Delta\right)-\partial_{j} \log \vartheta\left(-P_{1}+g P_{\infty}+\Delta\right)\right\} \zeta_{j}(y) \\
& \quad+\sum_{j=1}^{g}\left\{\partial_{j} \log \vartheta\left(-P_{1}+g P_{0}+\Delta\right)-\partial_{j} \log \vartheta\left(-P_{\infty}+g P_{0}+\Delta\right)\right\} \zeta_{j}(y),
\end{aligned}
$$

as claimed.

For $R(y)<R(x)$ we find that

$$
\begin{aligned}
& \sum_{n=-\infty}^{-(g+1)} f_{n}^{(0)}(x) f_{-n}^{(1)}(y) \\
& \quad=-\omega_{P_{\infty}-x}(y)-\sum_{j=1}^{g}\left\{\partial_{j} \log \vartheta\left(-x+g P_{0}+\Delta\right)-\partial_{j} \log \vartheta\left(-P_{\infty}+g P_{0}+\Delta\right)\right\} \zeta_{j}(y),
\end{aligned}
$$

and

$$
\begin{aligned}
\sum_{n=-g}^{-1} f_{n}^{(0)}(x) f_{-n}^{(1)}(y)= & \sum_{j=1}^{g}\left\{\partial_{j} \log \vartheta\left(-x+g P_{0}+\Delta\right)-\partial_{j} \log \vartheta\left(-P_{1}+g P_{0}+\Delta\right)\right\} \zeta_{j}(y) \\
& -\sum_{j=1}^{g}\left\{\partial_{j} \log \vartheta\left(-x+g P_{\infty}+\Delta\right)-\partial_{j} \log \vartheta\left(-P_{1}+g P_{\infty}+\vartheta\right)\right\} \zeta_{j}(y) .
\end{aligned}
$$

As a consequence,

$$
\begin{aligned}
& -\sum_{n \leqq-1} f_{n}^{(0)}(x) f_{-n}^{(1)}(y)=\omega_{P_{\infty}-x}(y) \\
& +\sum_{j=1}^{g}\left\{\partial_{j} \log \vartheta\left(-x+g P_{\infty}+\Delta\right)-\partial_{j} \log \vartheta\left(-P_{1}+g P_{\infty}+\Delta\right)\right\} \zeta_{j}(y) \\
& \quad+\sum_{j=1}^{g}\left\{\partial_{j} \log \vartheta\left(-P_{1}+g P_{0}+\Delta\right)-\partial_{j} \log \vartheta\left(-P_{\infty}+g P_{0}+\Delta\right)\right\} \zeta_{j}(y)
\end{aligned}
$$

in accordance with (IV.2). 
To prove (IV.3) we note that $K^{(1)}(x, y)=-K^{(0)}(y, x)+f_{0}^{(1)}(x)$. The proof of Theorem IV.2 is complete.

Corollary IV.3. $K^{(h)}(x, y)$ has a first-order pole at $x=y\left(\neq P_{0}, P_{\infty}\right)$. In the notation of (II.13),

$$
K^{(h)}(x, y)=\frac{1}{t(y)-t(x)}(1+O(t(x)-t(y)))(d t(x))^{h}(d t(y))^{1-h} .
$$

Definition IV.4. The $(h, 1-h)$-form $K^{(h)}(x, y)$ is called the Cauchy kernel of weight $h$.

The following theorem is the central result of this section. It states that any $h$-form $\omega$ holomorphic in an annulus $A(r ; R)$ can be expanded in a convergent Laurent series.

Theorem IV.5. Let $\omega \in \Gamma\left(S, \mathcal{O}^{h}\right)$ be holomorphic for $x \in A(r ; A)$ and continuous on $\bar{A}(r ; R)$. Then

$$
\omega(x)=\sum_{n \in \mathbb{Z}} \alpha_{n}^{(h)} f_{n}^{(h)}(x)
$$

where

$$
\alpha_{n}^{(h)}=(2 \pi i)^{-1} \int_{\gamma(\rho)} f_{-n}^{(1-h)}(x) \omega(x)
$$

for any $r<\rho<R$. The series (IV.9) converges almost uniformly on $A(r ; R)$. The $\alpha_{n}^{(h)}$ obey the Cauchy-type bounds

$$
\left|\alpha_{n}^{(h)}\right| \leqq \begin{cases}C M^{1} A_{0}^{(n)}(B \rho)^{-n+1}(1+B \rho)^{g-1}, & h=1, \quad 1 \leqq n \leqq g, \\ C M^{0} A_{1}^{(n)}(B \rho)^{-n}(1+B \rho)^{-g-1}, & h=0, \quad-g \leqq n \leqq-1, \\ C M^{0} A_{0}^{(1)}, & h=0, \quad n=0, \\ C M^{1} B \rho(1+B \rho)^{-2}, & h=1, \quad n=0, \\ C M^{h} A_{h}^{(n)}(B \rho)^{-n+h}(1+B \rho)^{-2 h+(2 h-1) g}, & \text { otherwise, }\end{cases}
$$

where $C<0$ is a constant, and where

$$
M^{h}:=\max _{x \in A(r ; \boldsymbol{R})}|\omega(x)| .
$$

Conversely, any series (IV.9) with coefficients $\alpha_{n}^{(h)}$ obeying bounds of the form (IV.11) defines an h-form holomorphic in a certain annulus.

Proof. We follow the lines of the proof of the standard Laurent expansion. As a consequence of Corollary IV.3,

$$
\omega(x)=(2 \pi i)^{-1} \int_{\partial A(r ; R)} K^{(h)}(x, y) \omega(y) .
$$

It follows from (IV.1) and Theorem IV.1 that

$$
\omega(x)=(2 \pi i)^{-1}\left(\int_{\gamma(R)}-\int_{\gamma(r)}\right) K^{(h)}(x, y) \omega(y)=\sum_{n \in \mathbb{Z}} \alpha_{n}^{(h)} f_{n}^{(h)}(x),
$$

with $\alpha_{n}^{(h)}$ given by (IV.10). Estimates (IV.11) are direct consequences of (III.12). The last statement of the theorem is clear. 


\section{The Small Denominators}

In this section we complete the proof of Theorem III.3, namely we establish the bounds (III.10). The main difficulty here is the fact that $\vartheta\left(P_{0}+D_{n}^{(h)}+(2 h-1) \Delta\right)$ occurring in the denominators of (III.7-9), although always nonzero, may approach zero as $n$ varies. Below we show, however, that for generic $P_{0}$ and $P_{\infty}$ the rate at which $\vartheta\left(P_{0}+D_{n}^{(h)}+(2 h-1) \Delta\right)$ approaches zero can be controlled.

To prove (III.10) we note that $f_{n}^{(h)}$ may be regarded as $h$-forms on a maximal compact domain $\mathscr{D} \subset \widehat{S}$ in the universal covering $\hat{S}$ of $S$ with a fixed global coordinate. Every factor in the definition of $f_{n}^{(h)}$ becomes a single-valued form and can be estimated separately. Independence of the estimate of the choice of $\mathscr{D}$ is a consequence of the single-valuedness of $f_{n}^{(h)}$ or $S$.

Clearly, for $x \in \mathscr{D}$,

$$
\begin{aligned}
& c_{1} \leqq\left|E\left(x, P_{0}\right)\right| \frac{1+R(x)}{R(x)} \leqq c_{2}, \\
& c_{3} \leqq\left|E\left(x, P_{\infty}\right)\right|(1+R(x)) \leqq c_{4}, \\
& c_{5} \leqq|\sigma(x)| \leqq c_{6},
\end{aligned}
$$

where $c_{j}, j=1, \ldots, 6$, are positive constants. Also, for $x \in \mathscr{D}$ and $D \in \operatorname{Div}(S)$,

$$
|\vartheta(x-D-\Delta)| \leqq c_{7}
$$

Therefore, (III.10) follows once we have found a bound from below on $\mid \vartheta\left(P_{0}+D_{n}^{(h)}+\right.$ $(2 h-1) \Delta) \mid$ (note that the singularity at $x=P_{1}$ in $f_{0}^{(1)}$ is removable).

Let $e \in J(S)$ be fixed and let $J(S) \ni \xi \rightarrow \theta(\xi-e)$ be a branch of the $\theta$-function. We consider the set $\Omega^{\varepsilon} \subset J(S)$ of points $f$ such that there exists a constant $0<c<\infty$ such that

$$
|\theta(n f-e)| \geqq c|n|^{-\left(2 g^{2}+g+\varepsilon\right)}, \quad n \in \mathbb{Z} .
$$

Let $\mu$ denote the Lebesgue measure on $J(S)$.

Theorem V.1. $\Omega^{\varepsilon}$ has full Lebesgue measure.

Proof. We use an idea taken from [A]. We show that the complement of $\Omega^{\varepsilon}$ has zero Lebesgue measure. The proof consists of two steps: First we estimate $\operatorname{dist}(n f, X)$, where $X$ is the set of zeroes of $\theta(\xi-e)$. We claim that the set of $f \in J(S)$ such that there exists $0<c<\infty$ with the property that

$$
\operatorname{dist}(n f, X) \geqq c|n|^{-(2 g+1+\varepsilon)}
$$

has full Lebesgue measure. Then we use the fact that $\theta(\xi-e)$ has zeroes of order at most $g$ to conclude the proof.

Since the complex structure of $J(S)$ is irrelevant for the present argument, it is no loss of generality to assume that $J(S)=\mathbb{R}^{2 g} / \mathbb{Z}^{2 g}$. Let $c>0$ be fixed. We define the set

$$
A_{c}=\bigcup_{n \in \mathbb{Z}\{\{0\}} \bigcup_{x \in X} \bigcup_{m \in \mathbb{Z}^{2 g}}\left\{f: \sum_{j=1}^{2 g}\left|n f_{j}-x_{j}-m_{j}\right| \leqq c|n|^{-(2 g+1+\varepsilon)}\right\} .
$$


Since $A_{c_{1}} \subset A_{c_{2}}$ if $c_{1}<c_{2}$ our assertion is proved once we have shown that

$$
\liminf _{c \rightarrow 0} \mu\left(A_{c}\right)=0 .
$$

Take $c$ sufficiently small. Since $-1 \leqq f_{j}-x_{j} / n \leqq 2$, it follows that $-n \leqq m_{j} \leqq 2 n$ in (V.5). Therefore

$$
\mu\left(A_{c}\right) \leqq 2 \sum_{n=1}^{\infty} \sum_{m:-n \leqq m_{j} \leqq 2 n} \mu\left(\bigcup_{x \in X}\left\{f:\left|f_{j}-\frac{x_{j}}{n}-\frac{m_{j}}{n}\right| \leqq c n^{-(2 g+2+\varepsilon)}\right\}\right) .
$$

We introduce the following notation. For $B \subset J(S)$,

$$
\begin{aligned}
B^{\varepsilon} & =\{\xi \in J(S): \operatorname{dist}(\xi, B) \leqq \varepsilon\}, \\
T_{a} B & =a+B, \quad a \in J(S), \\
\frac{1}{n} B & =\{\xi \in J(S): n \xi \in B\}, \quad 1 \leqq n \in \mathbb{Z} .
\end{aligned}
$$

Lemma V.2. Let $B$ be a measurable subset of $J(S)$. Then

(i) $\mu\left(B^{\varepsilon}\right)=O(\varepsilon)$, if $B$ is a closed submanifold of codimension 1,

(ii) $\mu\left(T_{a} B\right)=\mu(B)$,

(iii) $\mu\left(\frac{1}{n} B\right) \leqq \mu(B)$

(iv) $T_{a} B^{\varepsilon}=\left(T_{a} B\right)^{\varepsilon}$,

(v) $T_{a} \frac{1}{n} B=\frac{1}{n} T_{n a} B$,

(vi) $\frac{1}{n} B^{\varepsilon}=\left(\frac{1}{n} B\right)^{\varepsilon / n}$.

As a consequence of the lemma and the fact that $X$ is a union of finitely many closed submanifolds,

$$
\begin{aligned}
\mu\left(A_{c}\right) & \leqq 2 \sum_{n=1}^{\infty} \sum_{m:-n \leqq m_{j} \leqq 2 n} \mu\left(T_{m_{J} / n}\left(\frac{1}{n} X^{c n^{-(2 g+1+\varepsilon)}}\right)\right) \\
& \leqq O(c) \sum_{j=1}^{\infty} \frac{n^{2 g}}{n^{2 g+1+\varepsilon}}=O(c) \rightarrow 0,
\end{aligned}
$$

which proves (V.6). We can now complete the proof of the theorem. Since (V.4) holds $\mu$-almost everywhere, and the zeroes of $\theta(\xi-e)$ are of order at most $g$,

$$
|\theta(n f-e)| \geqq O(1)|n|^{-\left(2 g^{2}+g+\varepsilon\right)}
$$

for almost all $f$.

Proof of Lemma V.2. Only (i), (iii) and (vi) require arguments.

(i) Locally $\mu$ can be written as $\mu_{B} \times \mu_{\perp}$, where $\mu_{B}$ is a measure on $B$ and $\mu_{\perp}$ is transversal to $B$. 
(ii) We have

$$
\frac{1}{n} B=\bigcup_{k \in \mathbb{Z}^{2 g_{\cdot}} 0 \leqq k_{j} \leqq n-1}\left\{\xi: \xi_{j}=\frac{\eta_{j}}{n}+\frac{k_{j}}{n}, \eta \in B\right\} .
$$

Therefore

$$
\mu\left(\frac{1}{n} B\right) \leqq n^{2 g} \mu\left(\left\{\xi \cdot \xi_{j}=\frac{\eta_{j}}{n}, \eta \in B\right\}\right)=\mu(B) .
$$

(vi) We infer from (V.11) that $\xi \in(1 / n) B^{\varepsilon}$, iff

$$
\xi=\frac{1}{n} \tilde{\eta}+\frac{1}{n} k, \quad \tilde{\eta} \in B^{\varepsilon}, \quad 0 \leqq k_{j} \leqq n-1,
$$

which is equivalent to

$$
\xi=\frac{1}{n} \eta+\frac{1}{n} k+\frac{1}{n} \eta^{\prime}, \quad \eta \in B, \quad \operatorname{dist}\left(\eta^{\prime}, 0\right) \leqq \varepsilon .
$$

This proves the claim.

We can now complete the proof of Theorem II.3. In fact, since $\phi$ is continuous in the metric $\gamma$ the estimates hold for generic $P_{0}, P_{1}$ and $P_{\infty}$.

\section{Remarks on the Multidimensional Case}

The considerations of the previous sections can easily be generalized to a multi-dimensional context. Let $S^{m}$ be the $m$-fold Cartesian power of $S$. We set

$\gamma(\mathbf{r}):=\underset{j=1}{\times} \gamma\left(r_{j}\right)$ and $A(\mathbf{r} ; \mathbf{R}):=\underset{j=1}{\times} A\left(r_{j} ; R_{j}\right)$, where $\mathbf{r}=\left(r_{1}, \ldots, r_{m}\right), \mathbf{R}=\left(R_{1}, \ldots, R_{m}\right)$, $r_{j}<R_{j}$. Finally, we set

$$
K^{(\mathbf{h})}(\mathbf{x}, \mathbf{y}):=\prod_{j=1}^{m} K^{h_{j}}\left(x_{j}, y_{j}\right)
$$

for $\mathbf{x}, \mathbf{y} \in S^{m}$, and $\mathbf{h} \in \mathbb{Z}^{m}$. The following proposition is a consequence of the preceding sections.

Proposition VI.1. Let $\omega$ be an $\mathbf{h}$-form holomorphic in $A(\mathbf{r} ; \mathbf{R})$ and continuous in $\bar{A}(r ; \mathbf{R})$. Then

$$
\omega(\mathbf{x})=(2 \pi i)^{-m} \int_{\gamma(\mathbf{R})-\gamma(\mathbf{r})} K^{(h)}(\mathbf{x}, \mathbf{y}) \omega(\mathbf{y})
$$

Clearly, this proposition allows us to generate multidimensional Laurent expansions with the corresponding estimates on the coefficients. We omit the details. 


\section{References}

[AGMV] Alvarez-Gaumé, L., Gomes, C., Moore, G., Vafa, C.: Strings in the operator formalism. Nucl. Phys. B303, 455-521 (1988)

[A] Arnold, V. I.: Small denominators. I. Mappings of the circumference onto itself. Izv. Akad. Nauk SSSR, Ser. Mat. 25, 21-86 (1961)

[BS] Behnke, H., Stein, K.: Entwicklung analytischer Funktionen auf Riemannischen Flächen. Math. Ann. 120, 430-461 (1949)

[BLLR] Bonora, L., Lugo, A., Matone, M., Russo, J.: A global operator formalism on higher genus Riemann surfaces: $b-c$ Systems, preprint, 1988

[F] Fay, J. D.: Theta Functions on Riemann Surfaces. Berlin, Heidelberg, New York: Springer 1973

[HCR] Hurwitz, A., Courant, R., Röhl, H.: Funktionentheorie. Berlin, Göttingen, Heidelberg, New York: Springer 1964

[KN1] Krichever, I. M., Novikov, S. P.: Algebras of Virasoro type, Riemann surfaces and structures of the theory of solitons. Funkts. Anal. Pril. 21, 126-142 (1987)

[KN2] Krichever, I. M., Novikov, S. P.: Virasoro type algebras, Riemann surfaces and strings in Minkowski space. Funkts. Anal. Pril. 21, 294-307 (1987)

[M] Mumford, D.: Tata lectures on theta II. Boston, Basel, Stuttgart: Birkhäuser 1984

[S] Sonoda, H.: Calculation of a propagator on a Riemann surface. Phys. Lett. B178, 390-394 (1986)

Communicated by A. Jaffe

Received February 15, 1989; in revised form April 27, 1989 
